01

\title{
Особенности двумерных бифуркаций при диссипативном туннелировании электронов в массивах Аи наночастиц
}

\author{
(C) М.Б. Семенов, ${ }^{1}$ В.Д. Кревчик, ${ }^{1}$ Д.О. Филатов, ${ }^{3}$ А.В. Шорохов, ${ }^{1,2,7,8}$ А.П. Шкуринов, ${ }^{4}$ П.В. Кревчик, ${ }^{1}$
} Y.H. Wang, ${ }^{5}$ T.R. Li, ${ }^{5}$ A.K. Malik, ${ }^{6}$ Д.A. Антонов, ${ }^{3}$ И.M. Семенов ${ }^{1}$

${ }^{1}$ Пензенский государственный университет, 440026 Пенза, Россия

${ }^{2}$ University of Jyväskylä,

Seminaarinkatu, Fl-40014, Finland

${ }^{3}$ Нижегородский государственный университет им. Н.И. Лобачевского, 603950 Нижний Новгород, Россия

${ }^{4}$ Институт проблем лазерных и информационных технологий РАН (ИПЛИТ РАН), 140700 Шатура, Московская обл., Россия

${ }^{5}$ Key Laboratory for Special Function Materials, School of Physical Science and Technology, Lanzhou University, 730000 Lanzhou, China

${ }^{6}$ Department of Physics, Multanimal Modi College Modinagar,

201204 Uttar Prasesh, India

${ }^{7}$ Мордовский государственный университет им. Н.П. Огарева, Институт фризики и химии, 430005 Саранск, Россия

${ }^{8}$ International Research Centre Mag Top, Institute of Physics, Polish Academy of Sciences, PL-02668 Warsaw, Poland e-mail: Misha29.02.1@gmail.com

Поступило в Редакцию 2 апреля 2020 г.

В окончательной редакции 2 апреля 2020 г.

Принято к публикации 2 апреля 2020 г.

В рамках $2 D$-теории диссипативного туннелирования в квазиклассическом приближении (разреженного газа „пар: инстантон-антиинстантон“) при конечной температуре в условиях внешнего электрического поля исследованы особенности туннельного транспорта для планарных структур с квантовыми точками из коллоидного золота, предположительно обладающими свойствами метаматериала. Экспериментально было показано, что в зависимости от расположения иглы кантилевера комбинированного атомно-силового и сканирующего туннельного микроскопа (AFM/STM), либо над единичной квантовой точкой, либо между двумя соседними квантовыми точками, наблюдались соответственно либо один, либо двойной эффект $2 D$-туннельных бифуркаций (в виде излома(ов) на туннельной BAX). Как показывает наша теоретическая модель, именно такой режим двойной бифуркации (двойного сглаженного излома на туннельной $\mathrm{BAX}$ ) связан с проявлением свойств метаматериала исследуемой структурой. Получено убедительное качественное согласие между экспериментальными ВАХ и полевой зависимостью вероятности $2 D$-диссипативного туннелирования в двух исследованных режимах с учетом наблюдаемых квантовых биений (осцилляций) в окрестности точек $2 D$ бифуркации.

Ключевые слова: $2 D$-бифуркации, квантовое туннелирование с диссипацией, совмещенный АСМ/СТМ, массивы Аu-наночастиц.

DOI: 10.21883/JTF.2020.11.49965.111-20

\section{Введение}

Эффекты $2 D$-туннельных бифуркаций для квазиодномерных и квазидвумерных матриц Аи-квантовых точек $(\mathrm{Au}-\mathrm{KT})$ в тонких диэлектрических пленках во внешнем электрическом поле были теоретически и экспериментально изучены методом туннельной („проводящей“6) атомно-силовой микроскопии (САFM). В случае квазиодномерных структур $\mathrm{Au}-\mathrm{KT}$ (с размером КТ $\sim 5 \mathrm{~nm})$ в диэлектрической пленке на туннельной BAX наблюдается единичный излом при положительной полярности смещения, соответствующий эффекту бифуркации $2 D$-туннелирования, ранее предсказанный нами теоретически [1], а затем этот эффект 2D-бифуркации был обнаружен на экспериментальных туннельных BAX контакта зонда с образцом в системе CAFM в исследуемых планарных структурах с $\mathrm{Au}-\mathrm{KT}$ при позиционировании острия АFM-зонда строго над единичной $\mathrm{Au}-\mathrm{KT}$. Получено качественное согласие экспериментальных ВАХ и теоретической полевой зависимости вероятности $2 D$-диссипативного туннелирования в модельном потенциале $2 D$-осциллятора для случая параллельного туннелирования в пределе слабой диссипации при конечной температуре во внешнем элек- 
трическом поле. В случае квазидвумерных структур с $\mathrm{Au}-\mathrm{KT}$ (с размерами Аu-KT от 2 до $5 \mathrm{~nm}$ ) на экспериментальной BAX была обнаружена пара изломов, соответствующих двойному эффекту бифуркаций $2 D$ туннелирования. Качественное согласие между экспериментальными ВАХ и теоретической полевой зависимостью для вероятности $2 D$-диссипативного туннелирования было получено для ситуации с эффективно „отрицательной“ относительной диэлектрической проницаемостью матрицы среды-термостата, содержащие $\mathrm{Au}-\mathrm{KT}$.

Первое упоминание об эффекте бифуркаций $2 D$ туннелирования было найдено в работе Ю.Н. Овчинникова и Б.И. Ивлева [2] при рассмотрении двумерных систем с взаимодействующими джозефсоновскими контактами. Авторы предположили, что этот эффект может наблюдаться в виде характерного излома в соответствующих туннельных ВАХ, но его наблюдение оказалось трудным из-за размытия этой особенности токовым шумом в окрестности точки бифуркации. Неустойчивый эффект 2D-бифуркаций для случая антипараллельного туннельного транспорта наблюдался как неустойчивый излом для планарной структуры соединений типа порфиринов, где взаимодействующие протоны туннелируют во встречных направлениях по параллельным координатам реакции при исследовании температурной зависимости соответствующей скорости реакции [3]. Теоретическое исследование эффекта бифуркаций $2 D$ туннелирования в $2 D$-потенциалах с различными топологиями было развито в работах В.А. Бендерского и соавторов [4].

Эффект бифуркаций 2D-туннелирования для параллельного переноса взаимодействующих частиц в пределе слабой диссипации с учетом режима квантовых биений в окрестности точки бифуркации, предсказанный нами в более ранней работе [1], наблюдался экспериментально в квазиодномерной матрице $\mathrm{Au}-\mathrm{KT}$ и во внешнем электрическом поле методом САFМ [5].

Разд. 1.2 посвящен недавно выполненному эксперименту по измерению туннельных ВАХ для квазидвумерных массивов $\mathrm{Au}-\mathrm{KT}$.

В разд. 2.1 и 2.2 приведен теоретический расчет вероятности $2 D$-диссипативного туннелирования с учетом $2 D$-мод бифуркации в модельном потенциале $2 D$ осциллятора при конечной температуре во внешнем электрическом поле в пределе слабой диссипации для обеих диэлектрических матриц (эффективно квазиодномерный случай (разд. 2.1) и случай $2 D$-структур $\mathrm{Au}-\mathrm{KT}$ в диэлектрических матрицах (разд. 2.2).

Разд. 3 дает сравнение полученных экспериментальных и теоретических результатов и сравнивает эффекты $2 D$-туннельных бифуркаций как для квазиодномерных, так и для квазидвумерных структур с Аu-КТ. Сделан качественный вывод о том, что можно экспериментально подтвердить теоретически предсказанный эффект $2 D$ туннельной бифуркации для исследуемых наноструктур.

\section{1. Экспериментальные исследования двумерных туннельных бифуркаций}

\section{1. Экспериментальные BAX для квазиодномерных структур Au-KT}

Овчинников Ю.Н. (ИТФ им. Л.Д. Ландау) в своей работе [6] показал, что при исследовании проводимости планарных структур гранулированных металлических пленок, где соседние гранулы туннельно связаны между собой, взаимодействие с диэлектрической матрицей термостатом (the heat-bath), обеспечивающее реальный переход в состояния, локализованные в „соседнем“ кластере, оказывается достаточно малым. Как следствие, туннельный ток в исследуемых системах можно рассматривать в пределе сравнительно „слабой“ диссипации, но достаточной для обеспечения „затухания“ двухъямного осцилляторного потенциала, используемого в предлагаемой модели. Кроме того, значительный вклад в туннельный ток может с экспоненциальной точностью оцениваться вероятностью туннелирования [7].

Рис. $1, a$ представляет экспериментальную схему исследований и отдельные характерные туннельные ВАХ, полученные в рамках коллаборации научно-исследовательского центра „Оптика туннельносвязанных наноструктур и наноинженерия поверхности“ на базе кафедры „Физика“ факультета приборостроения, информационных технологий и электроники политехнического института Пензенского государственного университета с НОЦ „Физика твердотельных наноструктур“ Нижегородского государственного университета им. Н.И. Лобачевского [5]. При фиксации туннельного тока между острием кантилевера АСМ зонда и ближайшим нанокластером $\mathrm{Au}-\mathrm{KT}$ представляется возможным, что из-за неоднородностей на поверхности диэлектрической пленки могут реализоваться параллельные близко расположенные каналы туннельного тока. Если латеральные размеры выступов оказываются меньшими размеров $\mathrm{Au}-\mathrm{KT}$, то при изменении напряжения смещения асимметрия потенциала вдоль координаты z (перпендикулярно подложке) изменяется, и возможно переключение каналов токопереноса: в зависимости от величины напряженности внешнего электрического поля, а также от температуры и относительной диэлектрической проницаемости матрицы - термостата при параллельном синхронном переносе кулоновски взаимодействующих частиц происходит либо устойчивый синхронный (перескок туннелирующими электронами по параллельным каналам точек с одинаковыми координатами в одинаковые моменты времени (или „синхронно“), либо асинхронный перенос. Переключение с одного режима на другой соответствует точке бифуркации, которая будет кратко описана теоретически в разд. 2.1.

На рис. 1, $b$ представлена одна из характерных особенностей ВАХ в виде излома, наблюдаемого при положительных напряжениях смещения, который, как предполагается, связан с изменением режима туннелирования 

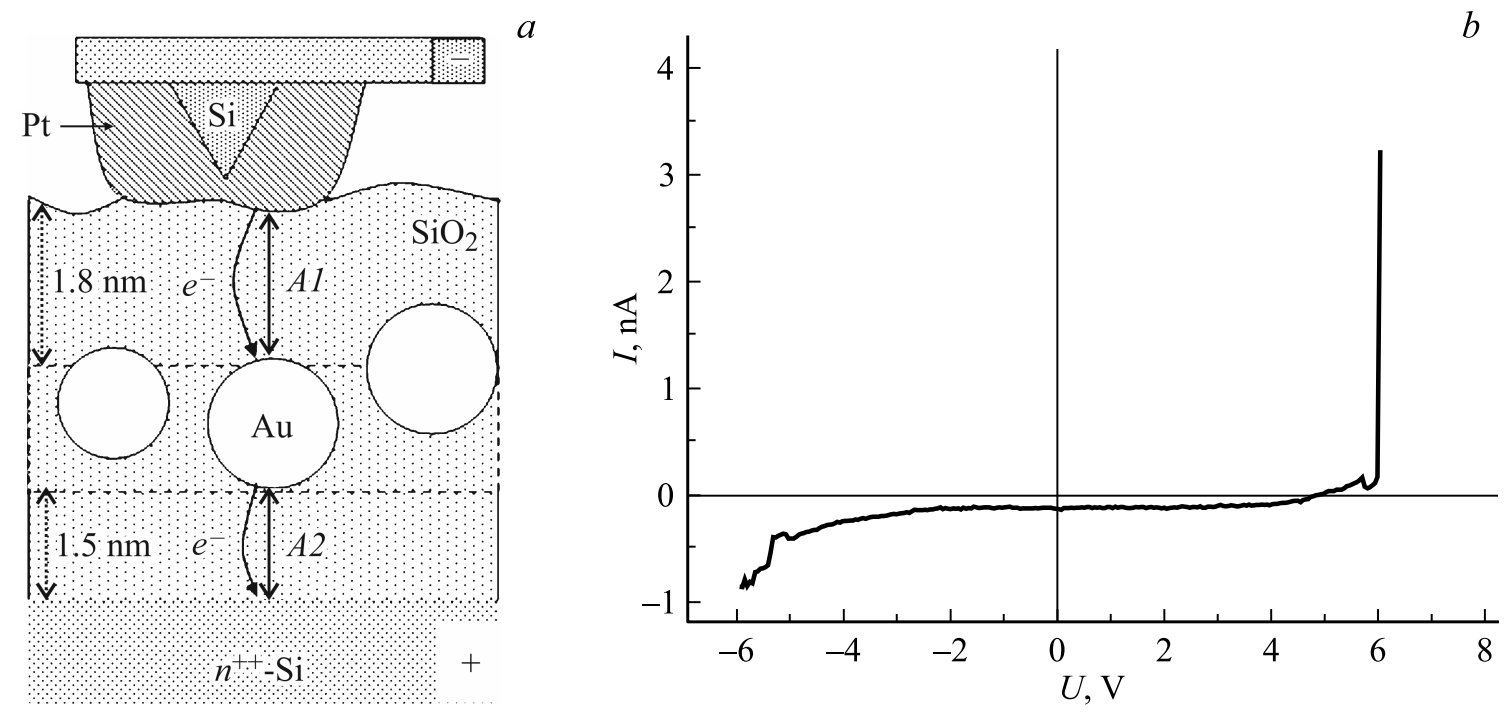

Рис. 1. $a$ - схематическое представление экспериментальной установки для изучения диссипативного туннелирования электронов через отдельные нанокластеры $\mathrm{Au}$, внедренные в туннельно-прозрачную пленку $\mathrm{SiO}_{2}$ на подложке $n^{+}-\mathrm{Si}$ методом CAFM. Al прозрачный потенциальный барьер „зонд-кластер“, $A 2$ - барьер „кластер-подложка“. $b$ - пример ВАХ контакта АСМ зонда к структуре $\mathrm{SiO}_{2}(1.5 \mathrm{~nm}) / \mathrm{SiO}_{2}: n c-\mathrm{Au}(1.6 \mathrm{~nm}) / \mathrm{SiO}_{2}(1.8 \mathrm{~nm}) / n^{+}-\mathrm{Si}(100)$.

вдоль параллельных каналов в асимметричном двумерном потенциале или наличием точки бифуркации, как описано в [1]. Вблизи этой точки бифуркации на ВАХ наблюдалась небольшая переходная область с отдельной особенностью, которая, вероятно, может соответствовать режиму квантовых биений, предсказанному в [1].

Наконец, при отрицательном напряжении наблюдался характерный одиночный пик, который, как описано в [7], связан с сингулярностью предэкспоненциального множителя в вероятности туннелирования в условиях, когда двухъямный осцилляторный потенциал модели становится симметричным с изменением внешнего электрического поля, которое влияет на величину параметра асимметрии $2 D$-осцилляторного потенциала.

Выполненный анализ этих теоретически и экспериментально изученных эффектов позволяет заключить, что можно экспериментально наблюдать устойчивые двумерные туннельные бифуркации с диссипацией, что является одним из основных результатов настоящей работы, и который качественно подтверждает гипотезу, высказанную в работах Э.Дж. Леггета, А.И. Ларкина, Ю.Н. Овчинникова и Б.И. Ивлева [3].

\section{2. Экспериментальные BAX для квазидвумерных структур Au-KT}

В рамках экспериментальной схемы, описанной в разд. 1.1, была реализована ситуация, когда туннельный ток формировался не через одну $\mathrm{Au}-\mathrm{KT}$ (квазиодномерный случай), а через две соседних Аu-КТ (квазидвумерный случай, рис. 2). Были получены следующие туннельные BAX (рис. 3), имеющие два „сглаженных“ излома.

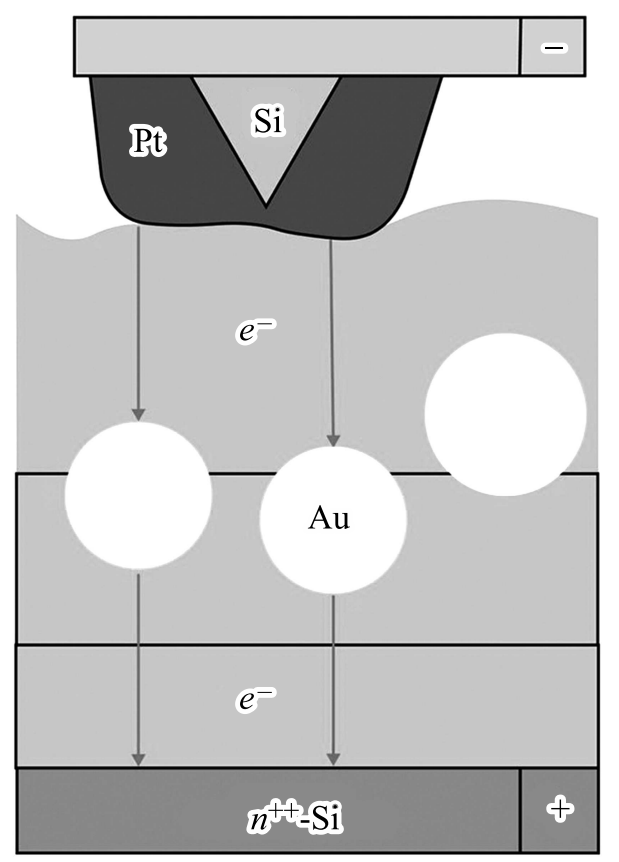

Рис. 2. Схематическое изображение экспериментальной установки для исследования диссипативного туннелирования электронов через $2 \mathrm{Au}-\mathrm{KT}$, внедренные в туннельно-прозрачную пленку $\mathrm{SiO}_{2}$ на подложке $n^{+}-\mathrm{Si}$ методом туннельной ACM.

На экспериментальной кривой рис. 3, $b$, помимо „сглаженных“ двойных изломов, наблюдались квантовые биения (осцилляции) в окрестности точек двумерных бифуркаций.

В разд. 3 будет проведено качественное сравнение туннельных ВАХ, приведенных на рис. 3, с полевой зави- 

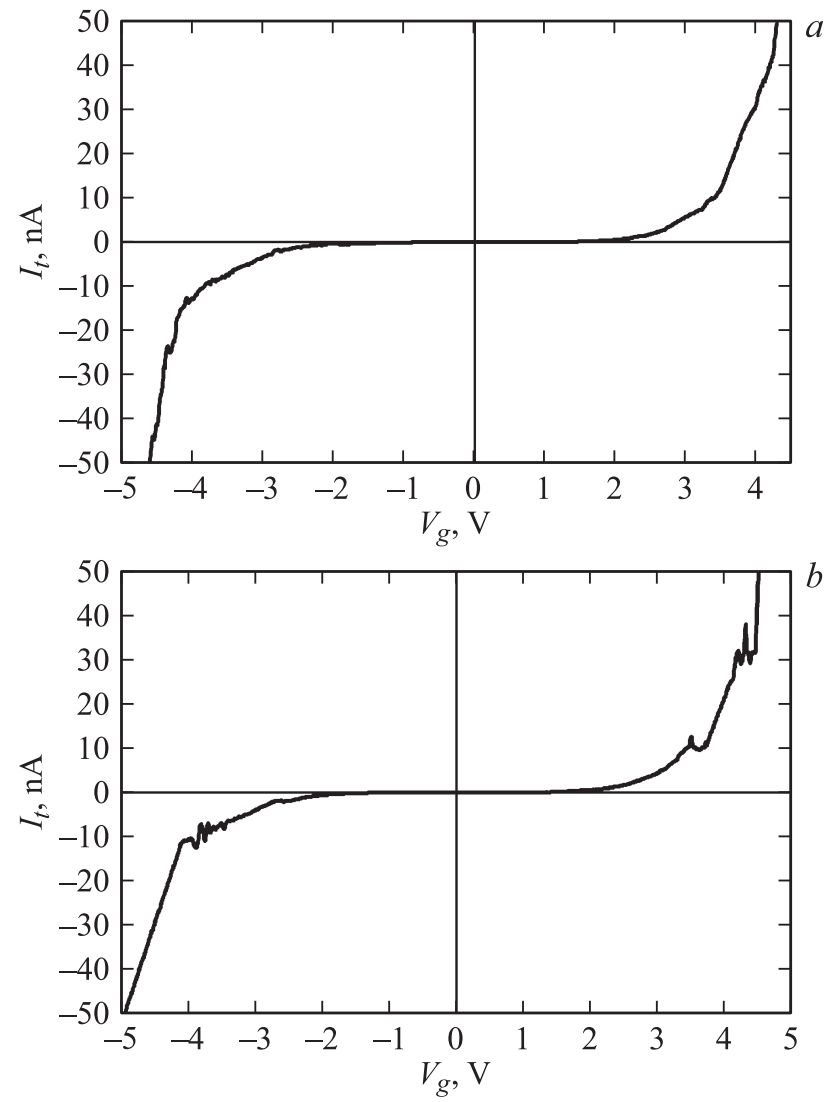

Рис. 3. Пример ВАX контакта АCM зонда к стуктуре $\mathrm{SiO}_{2}(1.5 \mathrm{~nm}) / \mathrm{SiO}_{2}: n c-\mathrm{Au}(1.6 \mathrm{~nm}) / \mathrm{SiO}_{2}(1.8 \mathrm{~nm}) / n^{+}-\mathrm{Si}(100)$, измененных при позиционировании АСМ зонда между двумя $\mathrm{Au}-\mathrm{KT}$ (рис. 2): $a-$ со „сглаженными“ двойными изломами (без квантовыми биений); $b-$ с квантовыми биениями.

симостью вероятности $2 D$-диссипативного параллельного туннелирования в случае квазидвумерной структуры.

\section{2. Сравнение теоретических полевых зависимостей вероятности 2D-диссипативного туннелирования для двух моделей матриц с экспериментальными туннельными BAX исследуемых планарных структур с квантовыми точками из коллоидного золота}

\section{1. Теоретический расчет}

$2 D$-бифуркационного режима в модели $2 D$-осцилляторного потенциала при конечной температуре во внешнем электрическом поле в пределе слабой диссипации для случая диэлектрической матрицы

Внешнее электрическое поле пропорционально меняет асимметрию двухъямного осцилляторного потенциала:

$$
\begin{aligned}
\tilde{U}(q) & =\frac{\omega_{0}^{2}}{2}\left(q-b_{0}\right)^{2} \theta(q) \\
& +\left[\frac{\omega_{0}^{2}}{2}\left(q+a_{0}\right)^{2}-\Delta I\right] \theta(-q)-|e| E q,
\end{aligned}
$$

где параметр $\Delta I=\frac{\omega_{0}^{2}}{2\left(a_{0}^{2}-b_{0}^{2}\right)}$ определяет исходную асимметрию потенциала в отсутствие внешнего электрического поля.

Для использования стандартной модели определения вероятности $2 D$-диссипативного туннелирования мы ввели следующие обозначения для перенормированного двухъямного осцилляторного потенциала во внешнем электрическом поле: $q_{1}=b=b_{0}+\frac{|e| E}{\omega_{0}^{2}}$, $q_{0}=\dot{a}=\dot{a}_{0}-\frac{|e| E}{\omega_{0}^{2}}$.

Для случая параллельно туннелирующих частиц выберем функцию $2 D$-потенциальной энергии $U_{1}\left(R_{1}, R_{2}\right)$ в следующей форме (см. [1]):

$$
\begin{aligned}
& U_{1}\left(R_{1}, R_{2}\right)=\frac{\omega^{2}\left(R_{1}+a\right)^{2}}{2} \theta\left(-R_{1}\right) \\
& +\left[-\Delta I+\frac{\omega^{2}\left(R_{1}-b\right)^{2}}{2}\right] \theta\left(R_{1}\right)+\frac{\omega^{2}\left(R_{2}+a\right)^{2}}{2} \theta\left(-R_{2}\right) \\
& +\left[-\Delta I+\frac{\omega^{2}\left(R_{2}-b\right)^{2}}{2}\right] \theta\left(R_{2}\right)-\frac{\alpha\left(R_{1}-R_{2}\right)^{2}}{2}
\end{aligned}
$$

где $\Delta I=\frac{1}{2} \omega^{2}\left(b^{2}-a^{2}\right)$ - является glqq теплотой реакции“ или параметром асимметрии потенциала; $\alpha-$ коэффициент взаимодействия между туннелирующими частицами, который в диполь-дипольном приближении зависит от величины относительной диэлектрической проницаемости среды - термостата (или диэлектрической матрицы), $(\alpha>0), R_{1,2}$ - параллельные координаты туннелирования частиц (вид этого $2 D$-потенциала представлен на рис. 4).

Принимая во внимание взаимодействие частиц, туннелирующих через параллельные каналы, реконструкция потенциала становится, по существу, двумерной. При определенном значении приложенного напряжения потенциал становится симметричным.

В рамках стандартной 2D-модели диссипативного туннелирования в пределах слабой диссипации [1] мы получим

$$
\begin{aligned}
& S\left\{R_{1}, R_{2}\right\}=\int_{-\beta / 2}^{\beta / 2} d \tau\left\{\frac{\dot{R}_{1}^{2}}{2}+\frac{\dot{R}_{2}^{2}}{2}+V\left(R_{1}, R_{2}\right)\right. \\
& \left.+\frac{1}{2} \int_{-\beta_{2}}^{\beta / 2} d \tau^{\prime} D\left(\tau-\tau^{\prime}\right)\left[R_{1}(\tau)+R_{2}(\tau)\right]\left[R_{1}\left(\tau^{\prime}\right)+R_{2}\left(\tau^{\prime}\right)\right]\right\},
\end{aligned}
$$

где

$$
D(\tau)=\frac{1}{\beta} \sum_{n=-\infty}^{\infty} D\left(v_{n}\right) e^{i} v_{n}^{\tau},
$$




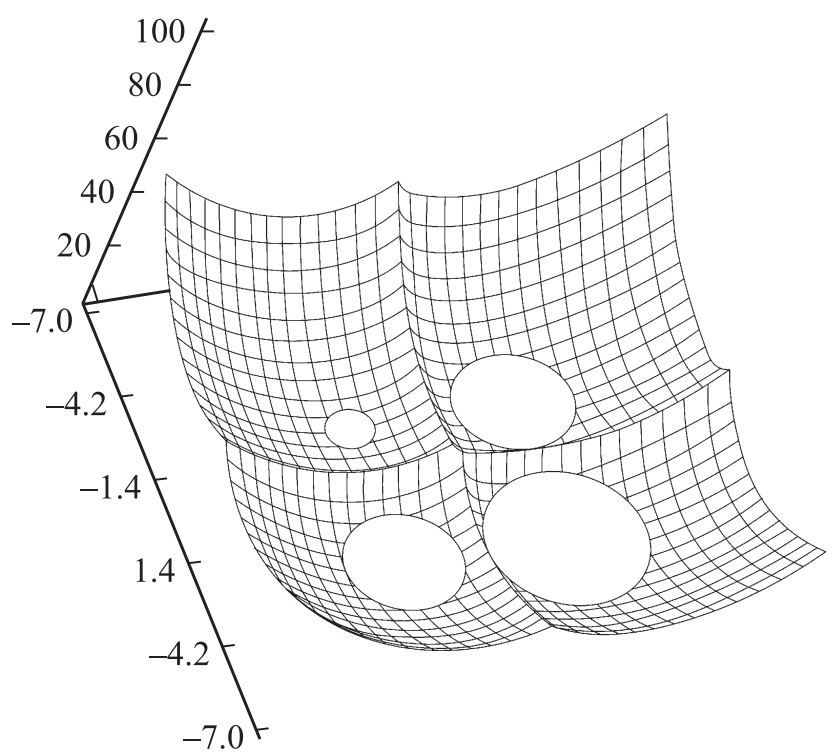

Рис. 4. Поверхность потенциальной энергии (2) как функция координат реакции для частиц, движущихся параллельно по координате $z$ (от зонда АСМ до ближайшей $\mathrm{Au}-\mathrm{KT}),(a=2$, $\left.b=2.5, \alpha^{*}=\frac{2 \alpha}{\omega^{2}} 0.0001\right)$.

$\beta=\frac{\hbar}{k_{\mathrm{B}} T}-$ обратная температура, $\left(\hbar, k_{\mathrm{B}}\right.$, а также массы частиц, как и ранее предполагаются равными единице), $v_{n}=2 \pi n / \beta-$ мацубаровские частоты; и, наконец, $D(\tau)$ - функции Грина фононов.

Двумерная квазиклассическая траектория (инстантон), которая минимизирует функционал действия, определяется из следующей системы квазиклассических уравнений движения [1]:

$$
\frac{\delta S}{\delta R_{1}}=0, \quad \frac{\delta S}{\delta R_{2}}=0
$$

Мы ищем решение системы этих уравнений в следующей форме:

$$
\begin{aligned}
& R_{1}(\tau)=\frac{1}{\beta} \sum_{n=-\infty}^{\infty} R_{n}^{(1)} e^{i v_{n} x} \\
& R_{2}(\tau)=\frac{1}{\beta} \sum_{n=-\infty}^{\infty} R_{n}^{(2)} e^{i v_{n} x}
\end{aligned}
$$

Решения системы уравнений (5) находятся в предположении одновременного начала туннелирования частиц. Времена $\tau_{1}$ и $\tau_{2}$ соответствуют моментам прохождения вершин барьеров частицами вдоль соответствующих координат реакции и могут быть найдены из следующих уравнений [1]:

$$
\begin{aligned}
& R_{1}\left(\tau_{1}\right)=0, \\
& R_{2}\left(\tau_{2}\right)=0 .
\end{aligned}
$$

В случае параллельного движения туннелирующих частиц (потенциальная энергия $\left.U_{1}\left(R_{1}, R_{2}\right),(2)\right)$, действие $S$ как функция параметров $\tau_{1}$ и $\tau_{2}$ имеет следующий вид [1]:

$$
\begin{aligned}
& S=2 a(b+a)\left(\tau_{1}+\tau_{2}\right) \omega^{2}-\frac{\omega^{2}(a+b)^{2}\left(\tau_{1}+\tau_{2}\right)^{2}}{\beta} \\
& -\frac{\omega^{4}(a+b)^{2}\left(\tau_{1}+\tau_{2}\right)^{2}}{\left(\omega^{2}-2 \alpha\right) \beta}-\frac{2 \omega^{4}(a+b)^{2}}{\beta} \\
& \times \sum_{n=1}^{\infty}\left\{\frac{\left(\sin v_{n} \tau_{1}+\sin v_{n} \tau_{2}\right)^{2}}{v_{n}^{2}\left(v_{n}^{2}+\omega^{2}+\xi_{n}\right)}+\frac{\left(\sin v_{n} \tau_{1}-\sin v_{n} \tau_{2}\right)^{2}}{v_{n}^{2}\left(v_{n}^{2}+\omega^{2}+2 \alpha\right)}\right\},
\end{aligned}
$$

где величины в пределе слабой диссипации могут быть приравнены нулю, т. е. если мы пренебрегаем взаимодействием туннелирующих частиц с осцилляторами среды.

Мы вводим обозначения: $\varepsilon=\varepsilon^{*} \omega=() \tau_{1}-\tau_{2} \omega$, $\tau=2 \tau^{*} \omega=\left(\tau_{1}+\tau_{2}\right) \omega, \quad \beta^{*}=\beta \omega / 2, \quad \alpha^{*}=2 \alpha / \omega^{2}$, $b^{*}=b / a, b \geq a$.

Если пренебречь взаимодействием с фононами (осцилляторами среды), тогда $\psi_{n}=0$, и действие $S(8)$ как функция параметров $\varepsilon=0$ и $\tau$ принимает вид

$$
\begin{aligned}
& S=(a+b) \omega \tau\left(2 a-\frac{a+b}{2}\left[1+\frac{1}{1-\alpha^{*}}\right]\right)+\frac{\omega(a+b)^{2}}{2} \\
& \times(\tau-|\varepsilon|) \frac{\alpha^{*}}{1-\alpha^{*}}-\frac{\omega(a+b)^{2}}{2}\left\{-\operatorname{cth} \beta^{*}+\frac{1}{\operatorname{sh} \beta^{*}}\right. \\
& \left.\times\left[\operatorname{ch}\left(\beta^{*}-\tau\right) \operatorname{ch} \varepsilon+\operatorname{ch}\left(\beta^{*}-\tau\right)-\operatorname{ch}\left(\beta^{*}-|\varepsilon|\right)\right]\right\} \\
& -\frac{\omega(a+b)^{2}}{2\left(1-\alpha^{*}\right)^{3 / 2}}\left\{-\operatorname{cth}\left(\beta^{*} \sqrt{1-\alpha^{*}}\right)+\frac{1}{\operatorname{sh}\left(\beta^{*} \sqrt{1-\alpha^{*}}\right)}\right. \\
& \times\left[\operatorname{ch}\left(\left(\beta^{*}-\tau\right) \sqrt{1-\alpha^{*}}\right) \operatorname{ch}\left(\varepsilon \sqrt{1-\alpha^{*}}\right)\right. \\
& \left.\left.-\left(\left(\beta^{*}-\tau\right) \sqrt{1-\alpha^{*}}\right)+\left(\left(\beta^{*}-|\varepsilon|\right) \sqrt{1-\alpha^{*}}\right)\right]\right\} .
\end{aligned}
$$

Мы перепишем соотношения (7) в виде

$$
\left\{\begin{array}{l}
\operatorname{sh} \varepsilon\left[\operatorname{ch} \tau \operatorname{cth} \beta^{*}-\operatorname{sh} \tau-\operatorname{cth} \beta^{*}\right]+\frac{1}{1-\alpha^{*}} \\
\times \operatorname{sh}\left(\varepsilon \sqrt{1-\alpha^{*}}\right)\left[\operatorname{ch}\left(\tau \sqrt{1-\alpha^{*}}\right) \operatorname{cth}\left(\beta^{*} \sqrt{1-\alpha^{*}}\right)\right. \\
\left.-\operatorname{sh}\left(\tau \sqrt{1-\alpha^{*}}\right)+\operatorname{cth}\left(\beta^{*} \sqrt{1-\alpha^{*}}\right)\right]=0, \\
3-\frac{4}{1+b^{*}}-\frac{1}{1-\alpha^{*}}+\operatorname{ch} \varepsilon\left[\operatorname{sh} \tau \operatorname{cth} \beta^{*}-\operatorname{ch} \tau-1\right] \\
+\operatorname{ch} \tau \operatorname{cth} \beta^{*}-\operatorname{ch} \tau+\frac{1}{1-\alpha^{*}} \operatorname{ch}\left(\varepsilon \sqrt{1-\alpha^{*}}\right), \\
{\left[\operatorname{sh}\left(\tau \sqrt{1-\alpha^{*}}\right) \operatorname{cth}\left(\beta^{*} \sqrt{1-\alpha^{*}}\right)\right.} \\
\left.-\operatorname{ch}\left(\tau \sqrt{1-\alpha^{*}}\right)+1\right]-\frac{1}{1-\alpha^{*}} \\
\times\left[\operatorname{sh}\left(\tau \sqrt{1-\alpha^{*}}\right) \operatorname{cth}\left(\beta^{*} \sqrt{1-\alpha^{*}}\right)\right. \\
\left.-\operatorname{ch}\left(\tau \sqrt{1-\alpha^{*}}\right)\right]=0 .
\end{array}\right.
$$


В дополнение к всегда существующему решению $\tau_{1}=\tau_{2}$, на рис. 5, $b, c$ появляются дополнительные решения (с увеличением параметра или понижением температуры). На рис. 5, $b$ показан момент (бифуркация) появления дополнительного решения, более предпочтительного по величине квазиклассического действия. На рис. 5, с уже есть два таких дополнительных решения, но только одно из них является предпочтительным по величине действия (в случае, когда эти решения близки, реализуется режим квантовых биений).

\section{2. Теоретический расчет}

$2 D$-бифуркационных мод в модельном потенциале $2 D$-осциллятора при конечной температуре во внешнем электрическом поле в пределе слабой диссипации для матрицы, обладающей свойствами метаматериала

В случае исследуемых квазидвумерных структур $\mathrm{Au}-$ КТ мы имеем дело с ситуацией, когда эти структуры могут иметь свойства метаматериала, т.е. относительная диэлектрическая проницаемость матрицы среды термостата эффективно становится „отрицательной“, и
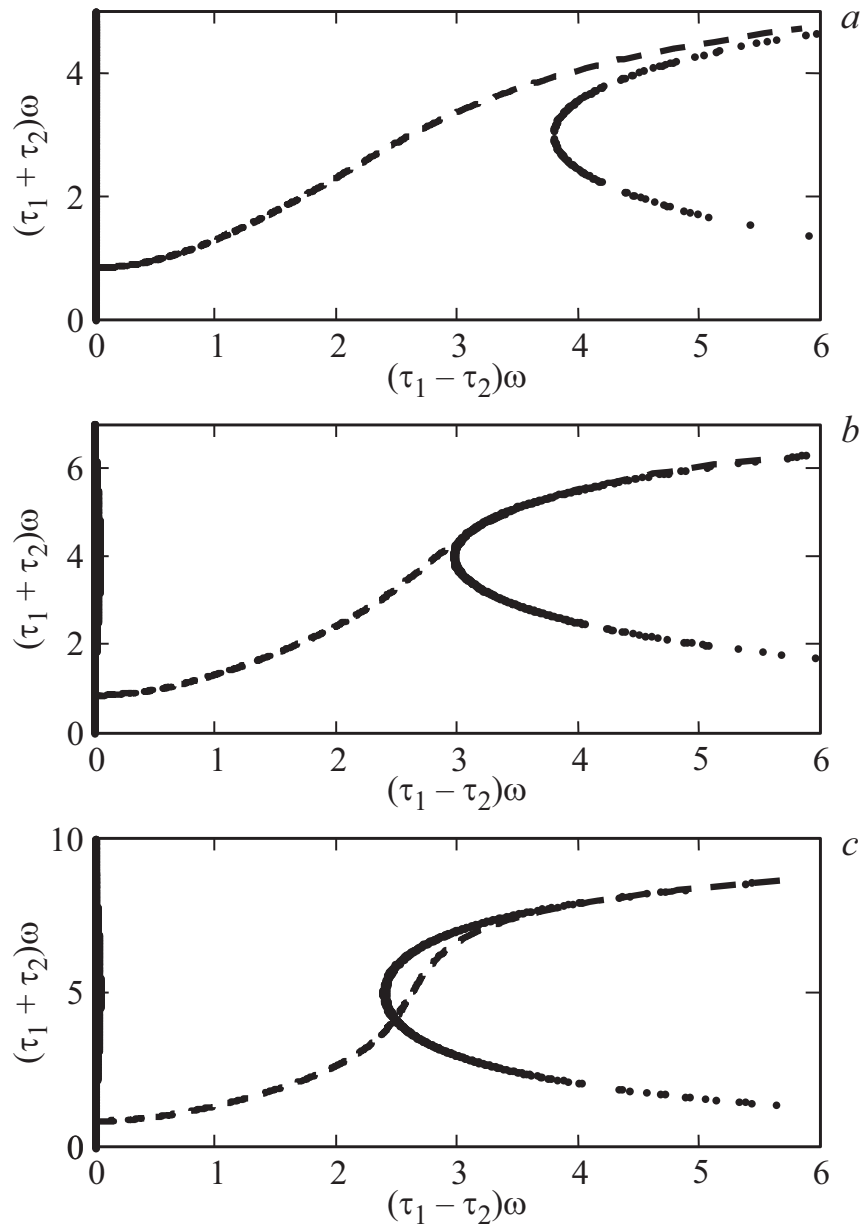

Рис. 5. Результаты численного решения системы трансцендентных уравнений (10).
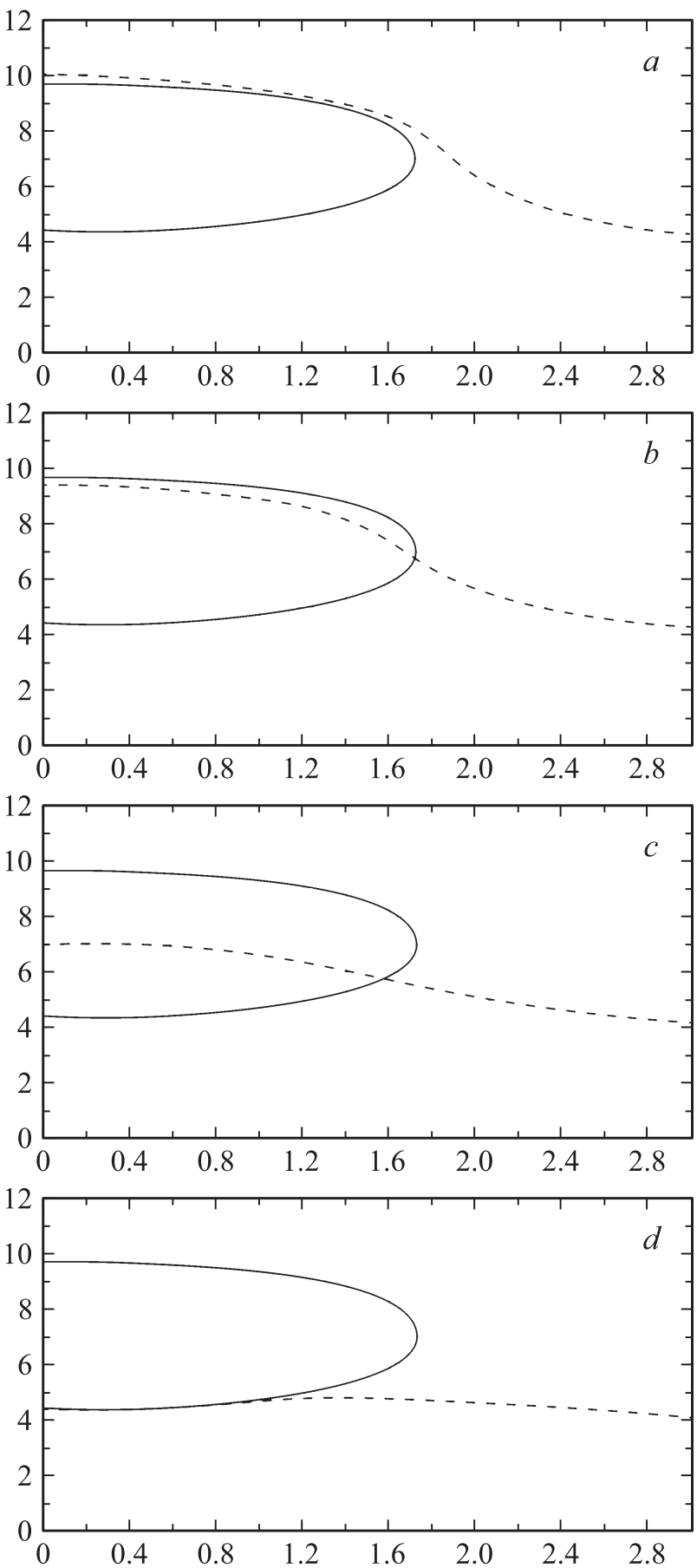

Рис. 6. Численное решение системы (10) для случая $\alpha<0$. Переход от $a$ к $d$ сопровождается увеличением напряженности электрического поля.

при этом коэффициент взаимодействия туннелирующих частиц в диполь-дипольном приближении также становится отрицательным $(\alpha<0)$. Как и в случае численного анализа системы уравнений (10), можно определить бифуркационные туннельные траектории для определенных значений температуры, либо параметра асимметрии потенциала (связанного с величиной внешнего электри- 


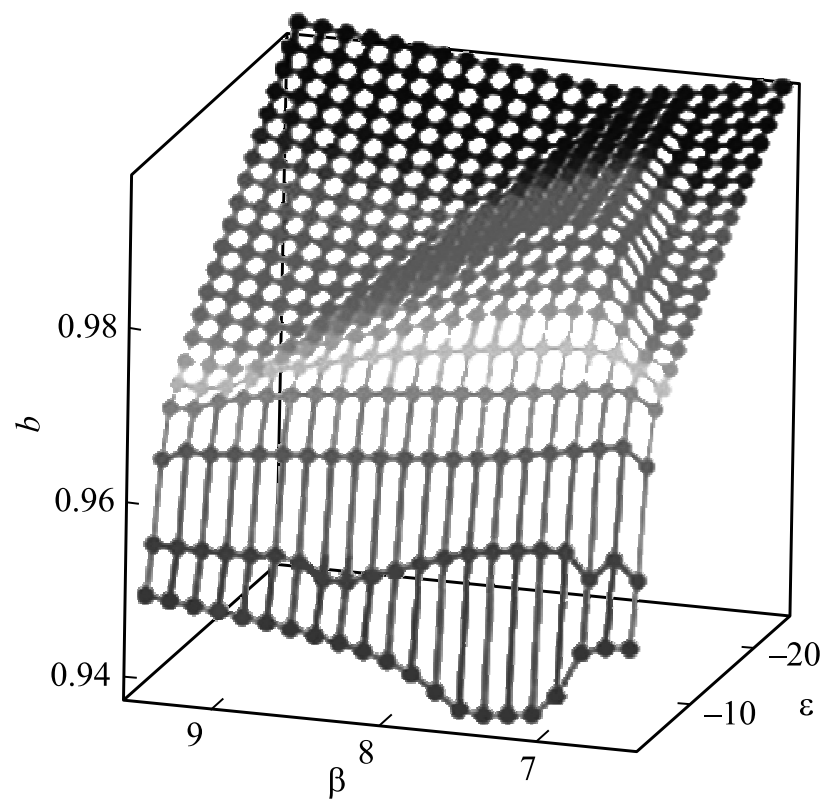

Рис. 7. „Фазовая“ диаграмма для реализации устойчивых бифуркаций тока $2 D$-туннелирования в матрице, обладающей свойствами метаматериала в зависимости от параметров управления: обратной температуры, напряженности электрического поля или значения (отрицательного) относительной диэлектрической проницаемости матрицы среды-термостата.

ческого поля), либо коэффициента взаимодействия $\alpha$ $(\alpha<0)$, который зависит от относительной диэлектрической проницаемости, отрицательной для метаматериалов. Численный анализ системы (10) также позволяет выявить тонкую структуру перехода в окрестности точки бифуркации, т. е. режим квантовых биений (осцилляций) для параллельного переноса туннелирующих частиц.

Этот численный анализ показан на рис. 6 (и, как легко убедиться, отличается от аналогичного результата, представленного на рис. 5).

В процессе исследования зависимости вероятности $2 D$-туннелирования от величины напряженности электрического поля выявлены области устойчивых двумерных бифуркаций. В окрестности начальной точки (как и в окрестности конечной точки) устойчивого режима двумерных бифуркаций реализуется механизм квантовых биений, где действуют конкурирующие механизмы синхронного и асинхронного туннельного переноса частиц. Выявлены области реализации устойчивого эффекта двумерных бифуркаций и численно проанализированы соответствующие границы существования двумерных бифуркаций при задании параметров управления (обратной температуры $\beta$, относительной диэлектрической проницаемости термостата $\varepsilon$ и параметра асимметрии для $2 D$-потенциала системы $b$, слабо нелинейно зависящего от величины напряженности внешнего электрического поля), а также проанализирована динамика изменения этих областей (рис. 7).
На рис. 7 представлена „фазовая“ диаграмма реализации устойчивого режима $2 D$-бифуркаций для туннельного тока (в случае квазидвумерной структуры $\mathrm{Au}-\mathrm{KT}$ ) в матрице в зависимости от параметров управления: обратной температуры, величины напряженности электрического поля (или параметра асимметрии потенциала) и величины (отрицательной) относительной диэлектрической проницаемости матрицы среды-термостата.

В результате исследована взаимосвязь напряженности внешнего электрического поля, при которой реализуются устойчивые двумерные бифуркации, от величины обратной температуры.

\section{3. Сравнительный анализ полученных экспериментально и теоретически эффектов 2D-туннельных бифуркаций как для случая квазиодномерных, так и квазидвумерных структур с Au-KT}

Как отмечалось в разд. 2.1, решение системы (10) позволяет идентифицировать бифуркацию $2 D$-туннельных траекторий при определенном значении параметра обратной температуры $\beta^{*}$, или параметра асимметрии $2 D$-потенциала $b^{*}=b / a$, связанного с величиной напряженности приложенного электрического поля, или коэффициента взаимодействия туннелирующих частиц $\alpha^{*}=2 \alpha / \omega^{2}$, (где $\alpha=\frac{e^{2}}{\varepsilon \varepsilon_{0} R_{0}^{3}}$, в частности, зависит от относительной диэлектрической проницаемости матрицы среды-термостата $\varepsilon$; проблема изучения двумерных бифуркаций с диссипацией при изменении параметра $\varepsilon$ может представлять отдельный интерес). Численный анализ системы (10) также позволяет выявить тонкую структуру перехода в окрестности точки бифуркации, а именно режим квантовых биений (осцилляций) для параллельного переноса туннелирующих частиц. В результате вероятность $2 D$-туннелирования с экспоненциальной точностью определяется как $\Gamma=\exp (-S)$, где $S$ задается выражением (9), с учетом решения системы (10). Поскольку мы планируем качественное сравнение полевой зависимости рассчитанной вероятности $2 D$-туннелирования с существующей экспериментальной туннельной BAX для системы ,игла кантилевера ACM зонда-нанокластеры $\mathrm{Au}-\mathrm{KT}$ (квазиодномерные структуры $\mathrm{Au}-\mathrm{KT}^{\text {“ }}$ (см. разд. 1.1)), то фактически необходимую полевую зависимость мы получаем через зависимость от параметра асимметрии $2 D$-потенциала. Результат сравнения этой теоретической кривой с экспериментальной BAX показан ниже на рис. 8. Необходимо учитывать, что мы рассматриваем две области изменения электрического поля: при положительном напряжении с реализацией $2 D$-бифуркационного режима и при отрицательном напряжении с достижением случая симметричного потенциала, который при реализации синхронного туннельного переноса по парал- 
лельным координатам дает в удвоенном предэкспоненциальном множителе наблюдаемую экспериментально особенность типа одиночного пика в этом случае.

Условия применимости рассматриваемой модели обусловлены квазиклассическим приближением разреженного газа „пар: инстантон-антиинстантон“ и обсуждались в [1-14]. В рассматриваемой модели кулоновские эффекты могут быть подавлены, если начальная энергия частицы в $\mathrm{Au}-\mathrm{KT}$ существенно превышает энергию кулоновского отталкивания: $U_{0} \gg \frac{e^{2}}{a_{0}+b_{0}}$.

Таким образом, обобщая результаты наших работ $[1,5]$, получено качественное сравнение теоретических кривых для вероятности диссипативного $2 D$ туннелирования как функции приложенного электрического поля с учетом точки бифуркации (при положительном напряжении) и наличия единичного пика в случае симметричного потенциала (с отрицательным напряжением) с отдельными экспериментальными ВАХ для системы „игла кантилевера АСМ зонда-нанокластеры Аu“, полученные группой соавторов из Нижегородского государственного университета им. Н.И. Лобачевского (в рамках существующей коллаборации научно-исследовательского центра „Оптика туннельно-связанных наноструктур и наноинженерия поверхности“ на базе кафедры Физика факультета приборостроения, информационных технологий и электроники политехнического института Пензенского государственного университета с НОЦ „Физика твердотельных наноструктур“ национального исследовательского Нижегородского государственного университета им. Н.И. Лобачевского). Эти результаты показаны на рис. 8.

В дополнение к довольно хорошему качественному согласию между теоретической и экспериментальной

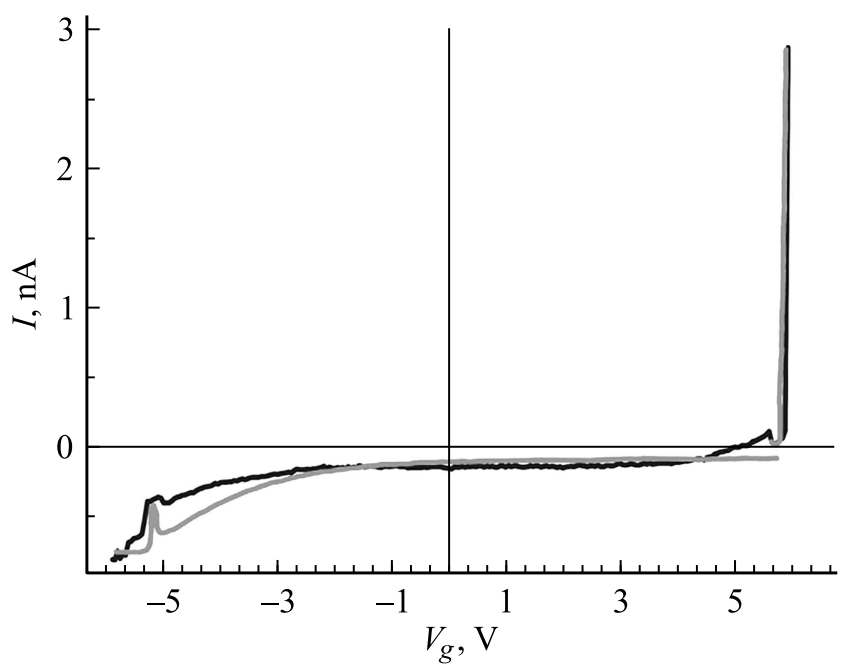

Рис. 8. Сравнение теоретической кривой (серая кривая) для вероятности двумерного диссипативного параллельного туннелирования с экспериментальной ВАХ, приведенной на рис. $1, b$ (черная кривая).
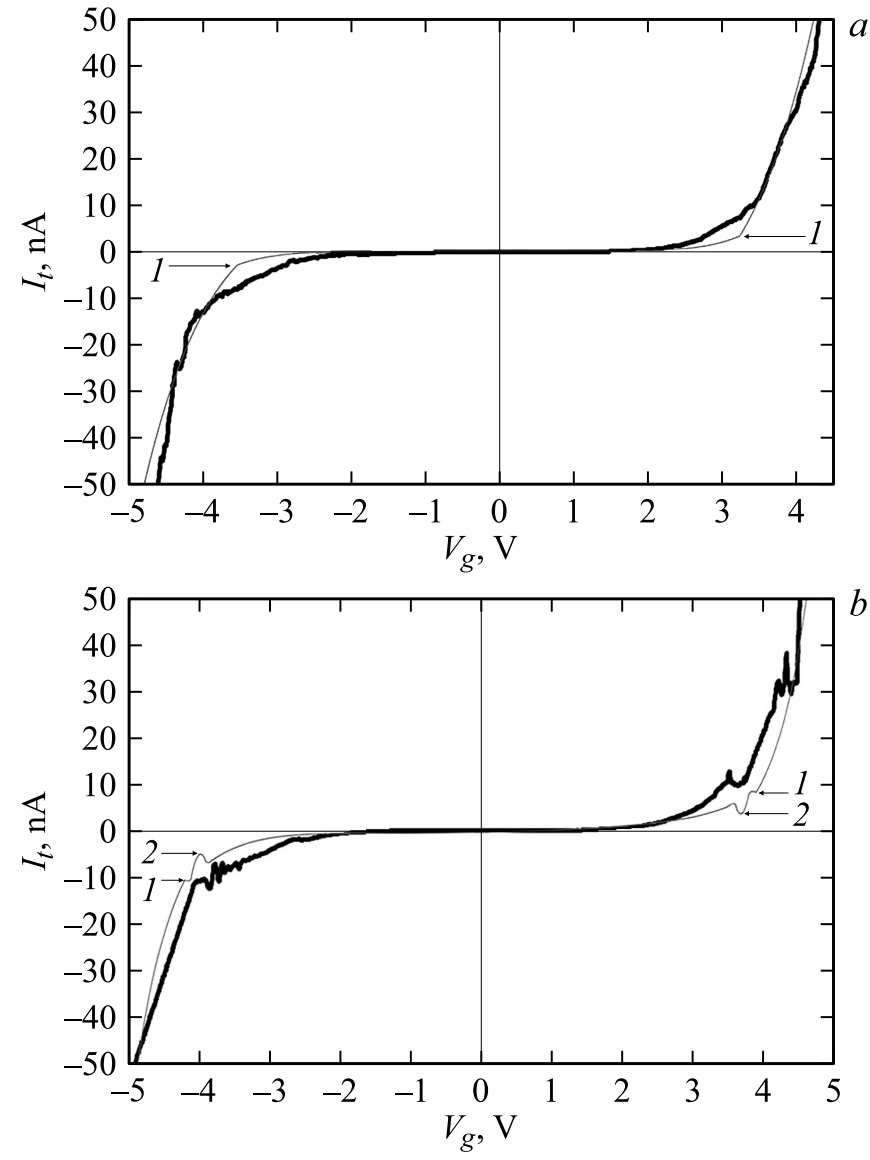

Рис. 9. Сравнение экспериментальной ВАХ (черная кривая) для квазидвумерных структур $\mathrm{Au}-\mathrm{KT}$, обладающих свойствами метаматериала (рис. 2,3), с полевой зависимостью вероятности $2 D$-диссипативного туннелирования (серая кривая), рассчитанной в разд. 2.2 в пределе слабой диссипации. $a-$ случай соответствует „сглаженным“ двойным изломам (1) или двумерным бифуркациям без режима квантовых биений. В случае (b) помимо „сглаженных“ двойных изломов (1) (или режима двойных $2 D$-бифуркаций), режим квантовых биений (2) также наблюдался в окрестности точек двумерных бифуркаций.

зависимостями (за исключением небольших переходных областей), результат этой работы позволяет сделать вывод, что устойчивая двумерная бифуркация (изменение режима туннелирования с синхронного на асинхронный), предсказанная в нашей более ранней работе [1], была найдена экспериментально. Вблизи этой точки (резкий излом в $\mathrm{BAX}$ ) небольшой локальный минимум может быть следствием квантовых биений, также описанного в [1] и учтенного в численном анализе, представленном на рис. 8. В случае квазидвумерных структур $\mathrm{Au}-\mathrm{KT}$, как отмечено в разд. 2.2, мы получаем на полевой зависимости вероятности $2 D$ диссипативного туннелирования не один, а два характерных излома. Результат сравнения этой полевой зависимости с экспериментальной ВАХ (рис. 3) показан на рис. 9. 


\section{Выводы}

Наука о квантовом туннелировании с диссипацией была развита в работах Э.Дж. Леггета, Я. Аффлека, П. Волыниса, А.И. Ларкина, Ю.Н. Овчинникова и других авторов [1-10]. Ранее в своем обзоре $[3,6,8]$ Э.Дж. Леггет поставил „сверхзадачу“ экспериментального наблюдения макроскопических квантовых эффектов диссипативного туннелирования. Возможности и развитие современной технологии наноструктур приблизили реализацию этой задачи. Мы надеемся, что настоящей работой мы сделаем наш скромный вклад в ее решение.

\section{Благодарности}

Авторы благодарят проф. Э.Дж. Леггета и Ю.Н. Овчинникова (который впервые вместе с Б.И. Ивлевым предсказал эффект бифуркаций $2 D$-туннелирования) за полезные обсуждения, а также А.В. Зенкевича и Ю.А. Матвеева (Национальный исследовательский университет „Московский физико-технический институт МФТИ“ ) за изготовление образцов для экспериментальных исследований.

Авторы благодарят ИПЛИТ РАН, ЦКП МГУ им. М.В. Ломоносова и НОЦ „Физика твердотельных наноструктур“ ННГУ им. Н.И. Лобачевского за помощь при выполнении экспериментальной части работы.

\section{Финансирование}

Работа выполнена при поддержке гранта Министерства науки и высшего образования РФ 0748-2020-0012 и гранта РФФИ 18-42-130007 p_a. The International Center for Interfacing Magnetism and Superconductivity with Topological Matter Mag Top is supported by the Foundation for Polish Science through the IRA Programme co-financed by EU within SG OP (Grant No. MAB/2017/1).

\section{Конфликт интересов}

Авторы заявляют, что у них нет конфликта интересов.

\section{Список литературы}

[1] Aringazin A.K., Dahnovsky Yu.I., Krevchik V.D., Semenov M.B., Ovchinnikov A.A, Yamamoto K. // Phys. Rev. B. 2003. Vol. 68. P. 155426 (1-12).

[2] Ivlev B.I., Ovchinnikov Yu.N. // JETP, 1987. Vol. 66. N 2. P. 378-383. [Zhurnal Éksperimental'noři Teoreticheskoř Fiziki, Vol. 93. 1987. P. 668-679.]

[3] Леггет Э.Джс. (гл. редактор), редакторы: Арынгазин А.К., Бендерский В.А., Дахновский Ю.И., Деккер Х., Жуковский В.Ч., Кревчик В.Д., Овчинников Ю.Н., Семенов М.Б., Тернов А.И., Ямамото К.; соавторы: Антонов Д.А., Веремьев В.А., Ветошкин Е.В., Волчихин В.И., Гак Л.Н., Гориков О.Н., Грозная Е.В., Грунин А.Б., Губина С.А., Зайцев Р.В., Ивлев Б.И., Йорк Джк.Т., Калдейра А.О., Кач, Е.И., Коалсон Р.Д., Кревчик П.В., Кудряшов Е.И., Лапшина М.А., Ларкин А.И., Овчинников А.А.,
Разумов А.В., Рудин В.А., Семенов О.Ф., Скибиикая Н.Ю., Смирнов Ю.Г., Суто А., Тромсдорб Г.П., Фальковский Л.А., Филатов Д.О., Чупрунов Е.В., Шенина М.Е. Управляемое диссипативное туннелирование. Туннельный транспорт в низкоразмерных системах. Коллективная монография, посвященная памяти академика РАН А.И. Ларкина (ИТФ им. Л.Д. Ландау) под редакцией Нобелевского лауреата по физике 2003, проф. Э.Дж. Леггета, Москва, „Физматлит“, 2011-2012, 496 cс.

[4] Benderskii V.A., Vetoshkin E.V., Trommsdorff H.P., Kats E.I. // Phys. Rev. E. 2003. Vol. 67. P. 026102.

[5] Жуковский В.Ч., Дахновский Ю.И., Горшков О.Н., Кревчик В.Д., Семенов М.Б., Смирнов Ю.Г., Чупрунов Е.В., Рудин В.А., Скибицкая Н.Ю., Кревчик П.В., Филатов Д.О., Антонов Д.А. Лапшина М.А., Шенина М.Е., Ямамото K. // Вестник Московского университета (сер. 3, Физика и Астрономия). 2009. N 5. 3-8 с.

[6] Leggett A.J. (main editor), editors: Dahnovsky Yu., Krevchik V.D., Krivnov V.Ya., Semenov M.B., Yamamoto K., coauthors: Aringazin A.K., Astakhova T.Yu., Back C., Balagurov B.Ya., Benderskii V.A., van den Brink Alec Maassen, Caldeira A.O., Coalson R.D., Danilov S.N., Dawson Wayne, Dekker H., Dmitriev D.V., Dzyaloshinsky I.E., Feigel'man M.V., Fernandez-Ramos Antonio, Ganichev S.D., Goldanskii V.I., Grunin A.B., Imamov E.Z., Ivlev B.I., , Kats E.I., Kudryashov E.I., Larkin A.I., Liao Jie-Lou, Margulis V.A., Marko A.A., Mayorov V.G., Menon Madhu, Mironov A.D., Moskalenko A.S., Nechaev S.K., Olkhovsky V.S., Ovchinnikov A.A., Ovchinnikova M.Ya., Ovchinnikov Yu.N., Perel V.I., Pollak Eli, Prettl W., Rakityansky S.A., Recami Erasmo, Ruda H., Shik A.Ya., Shorokhov A.V., Siebrand W., Sollinger M., Smedarchina Z., Suzuki Kazuo, Trommsdorff Y.P., Trushin M.P., Vasilyev O.A., Veremyev V.A., Vetoshkin E.V., Vinogradov G.A., Vinokur V.M., Yassievich I.N., York J.T., Zabrodin A.V., Zimmermann J., Zhukovsky V.Ch. Transfer processes in low-dimensional systems (in memory of A.A. Ovchinnikov and A.I. Larkin): - UT Research Institute Press, Tokyo, Japan, 2005. 690 p.

[7] Жуковский В.Ч., Горшков О.Н., Кревчик В.Д., Семенов М.Б., Грозная Е.В., Филатов Д.О., Антонов Д.А. // Вестник Московского университета (сер. 3, Физика и Астрономия). 2009. N 1. 27-31 с.

[8] Caldeira A.O., Leggett A.J. // Ann. Phys. 1983. Vol. 149. N 2. P. 374-456.

[9] Larkin A.I., Ovchinnikov Yu.N. // Phys. Rev. B. 1983. Vol. 28. N 11. P. 6281-6285.

[10] Ovchinnikov Yu.N // JETP. 2007. Vol. 131. N 2. P. 286-290.

[11] Filatov D., Guseinov D., Antonov I., Kasatkin A., Gorshkov O. // Royal Society Chem. Adv. 2014. N 4. P. 57337-57342.

[12] Engheta N., Ziolkowski R.W. Metamaterials: Physics and Engineering Explorations. John Wiley \& Sons \& IEEE Press, 2006.

[13] Valentine J., Zhang Sh., Zentgraf T., Ulin-Avila E., Genov D., Bartal G., Zhang X. // Nature. 2008. Vol. 455. N 7211. P. 376-379.

[14] National Science Review, Special issue „Metamaterials Collection“. 2018. N 5. 